\title{
Complete Remission of del(5q) Myelodysplastic Syndrome after 7 Days of Lenalidomide Therapy Gives an Alert!
}

\author{
Hassan A. Al-Jafar ${ }^{a} \quad$ Salih Al-Azmi ${ }^{\text {b J.A. Qassem }}{ }^{c}$ \\ Eman A. Hasan ${ }^{d}$ Arwa Alduaij $^{\mathrm{e}}$ \\ ${ }^{\mathrm{a}}$ Haematology Department, ${ }^{\mathrm{b}}$ Respiratory Department, ${ }^{\mathrm{c}}$ Internal Medicine \\ Department, ${ }^{\mathrm{d}}$ Rheumatology Department, and ${ }^{\mathrm{e}}$ Medical Casualty Department, \\ Amiri Hospital, Kuwait City, Kuwait
}

\section{Key Words}

Lenalidomide $\cdot$ Myelodysplastic syndrome $\cdot 5 q$ del $\cdot$ Blood transfusion $\cdot$ Remission

\begin{abstract}
Myelodysplastic syndrome (MDS) refers to a group of haematological, monoclonal disorders. A 50-year-old woman was diagnosed with MDS $5 q$ deletion syndrome [del( $5 q)]$, becoming dependent on blood transfusion after long-term treatment with cytotoxic drugs for chronic scleritis. Lenalidomide therapy $(10 \mathrm{mg} /$ day) led to profound pancytopaenia, followed by recovery of her blood cell counts. A cytogenetic study, repeated 4 months after lenalidomide treatment, revealed complete remission after only 1 week of lenalidomide therapy. Lenalidomide was approved for low- and intermediate-1-risk MDS, where it normalises platelet counts and induces haematological and cytogenetic remission. This patient has remained transfusion independent for 3 years by continuing on a minimal maintenance dose of lenalidomide. Starting MDS patients on lenalidomide has to be done cautiously or with only $5 \mathrm{mg} /$ day because of the potentially high sensitivity of the stem cells to this immunomodulatory agent in MDS patients.
\end{abstract}

\section{Introduction}

Myelodysplastic syndrome (MDS) is a group of conditions involving a heterogeneous group of clonal, neoplastic stem cells. The conditions are often characterised by a hypercellular bone marrow, but hypocellularity may also be observed [1]. MDS is also considered to be a premalignant condition that may progress to bone marrow failure. MDS patients usually present with abnormal blood cell morphological features arising 
because of impaired maturation and peripheral blood cytopaenias in one or more cell lineages. These abnormalities result from ineffective blood cell production. The diagnosis and treatment of MDS remains a challenging task in clinical haematology.

MDS can be classified as one of two types. These are de novo, primary MDS and secondary MDS, which is due to aggressive treatment of other cancers or exposure to irradiation, alkylating agents, or topoisomerase II inhibitors; secondary MDS may also occur in patients who have been heavily pretreated prior to receiving an autologous bone marrow transplant [2]. The French-American-British (FAB) classification, which served as the gold standard for morphologic classifications over more than 2 decades, has largely been replaced by the World Health Organisation (WHO) classification. Risk stratification traditionally applies widespread, clinically available parameters, such as the percentage of marrow blasts, karyotype, and number of cytopaenias, as calculated by the widely adopted International Prognostic Scoring System (IPSS). Patients, based on their IPSS scores, may be stratified into a 'lower-risk' group, comprised of those who are in the low- or intermediate-1-risk category, or into a 'higher-risk' group, comprised of patients with IPSS scores that put them in the intermediate-2- or high-risk categories [3]. Another prognostic factor is the patient's blood transfusion history; iron overload, resulting from more frequent transfusions, increases the risk associated with MDS [4].

Chromosome $5 q$ deletions [del(5q)] are common abnormalities associated with MDS. The chromosomal deletion in patients with del(5q) MDS is of variable size, with a predominance of large, 5q13-33 deletions. However, the most commonly deleted chromosomal region is 5q31-32 [5]. There are several clinical and biological features associated with patients with del(5q) MDS, which include a preponderance of female sex, severe anaemia, pronounced macrocytosis, normal or moderately decreased leukocyte counts, normal or moderately increased platelet counts, rare acute myeloblastic leukaemia (AML) transformations (10\%), and prolonged survival. Bone marrow features in these patients include characteristic dysmegakaryopoiesis (large monolobulated megakaryocytes with eccentric nuclei), and no or moderate blast excess (restricted to $<5 \%$ marrow blasts according to the WHO classification) [6].

We present a patient who showed an unexpected response to lenalidomide, becoming profoundly pancytopaenic. This case is being presented to highlight the potential outcomes if a patient is not closely followed during outpatient follow-up visits when on lenalidomide therapy.

\section{Case Presentation}

A 50-year-old woman had been diagnosed 11 years previously with autoimmune scleritis that resulted in pain and conjunctival congestion. Since that time, she had undergone treatment with several immunosuppressive drugs, including prednisolone, methotrexate, imuran, mycophenolate mofetil, cyclosporine, and adalimumab. After 9 months of adalimumab (Humira ${ }^{\circledR}$; Abbott Laboratories, North Chicago, Ill., USA) treatment, she started to complain of weakness, pallor, low blood count, and progressive anaemia. The patient became transfusion dependent and was referred to our haematology outpatient department. Investigations conducted in this department revealed macrocytic anaemia with hypogranular granulocytes; pseudo-Pelger-Huet anomaly myelodysplastic features; and normal levels of platelets, serum vitamin B12, folate, and serum ferritin. Her complete blood count (CBC) on presentation, but after a transfusion, included a white blood cell (WBC) count of $3.0 \times 10^{9} / \mathrm{l}$, a haemoglobin level of $73 \mathrm{~g} / \mathrm{l}$, a mean corpuscular volume of $108 \mathrm{fl}$, and a platelet count of $225 \times 10^{9} / \mathrm{l}$; the patient's haemoglobin level three weeks later fell to $50 \mathrm{~g} / \mathrm{l}$. Further, a bone marrow 
aspiration was normocellular but revealed the presence of dysplastic red blood cell precursors, elevated numbers of hypolobulated megakaryocytes, $4 \%$ blast cells, and an absence of ring sideroblasts; a cytogenetic karyotype study revealed del(5q) MDS (fig. 1 and fig. 2 ).

Lenalidomide (10 mg/day) was prescribed. After 1 week, the patient returned for a CBC recheck. At this point, the patient was admitted to the hospital because of a low cell count and chest infection. Her blood count continued to drop, in spite of termination of her lenalidomide treatment. After a few days, her blood counts reached very low levels, with a WBC count of $0.9 \times 10^{9} / \mathrm{l}$, a haemoglobin level of $75 \mathrm{~g} / \mathrm{l}$, and a platelet count of $4 \times 10^{9} / \mathrm{l}$. She required recurrent red blood cell and platelet transfusions and was started on a broad-spectrum antibiotic because of her severe chest infection.

After 3 weeks of profound pancytopaenia, the patient's blood counts slowly started to regenerate, allowing her to be discharged from the hospital after 4 weeks. Four weeks after discharge, her blood count returned to the normal level and her mean corpuscular volume (MCV) started to rise again to more than the normal reference value so she began taking a maintenance dose of lenalidomide $(5 \mathrm{mg} /$ day). Three months after discharge, the patient underwent a second cytogenetic examination that revealed a normal karyotype (fig. 2). Therefore, the patient was put on a reduced maintenance dose of lenalidomide ( $5 \mathrm{mg}$, twice per week). This minimal maintenance dose has successfully kept the patient in remission. However, the dose is regularly monitored by checking her MCV; if it rises, the dose is increased to adjust the MCV to the normal value, the doses of lenalidomide used were not more than $5 \mathrm{mg} /$ day.

\section{Discussion}

Lenalidomide is an immunomodulatory drug with erythropoietic activity in MDS that are mainly karyotype dependent, providing a benefit to patients who otherwise would not be expected to benefit from recombinant erythropoietin therapy. Long-term outcome data indicate that a cytogenetic response to lenalidomide therapy might offer a survival advantage, compared with cytogenetic nonresponders, and it also reduces the risk for the development of AML transformation, among lower-risk, transfusiondependent MDS patients without del(5q) [7]. Lenalidomide causes a reduction in the generation of inflammatory cytokines and marrow microvessel density and also improves the function of the primitive haematopoietic progenitor cells. An improvement in cytologic dysplasia is common in patients with del(5q) but was infrequent in erythroid-responding patients without del(5q). These findings indicate that lenalidomide promotes erythropoiesis among lower-risk MDS patients via 2 apparently distinct mechanisms of action, namely, suppression of the ineffective del(5q) clone and promotion of effective erythropoiesis in non-del(5q) MDS progenitor cells. The most common adverse reactions include dose-dependent neutropaenia and thrombocytopaenia, events that are more pronounced in patients with del(5q) in whom early suppression of the clone is expected [8]. Lenalidomide is also an analogue of thalidomide. Therefore, although it has not been demonstrated to have teratogenic effects in animal models, women taking the medication should avoid pregnancy [9].

Lenalidomide is a highly effective drug for the treatment of del(5q) MDS, and showed a significant response in the present case. The reason for the large response may have been that the del( $5 q)$ clone, in this case, was highly sensitive to lenalidomide, resulting in the rapid clearing of the clone from the patient's marrow and a resultant profound pancytopaenia. Lenalidomide appears to specifically target the del(5q) clone, suggesting that it may have a direct effect on the course of the disease. In particular, the drug appears to be especially active in patients with intermediate-2- and high-risk MDS with del(5q), i.e. those patients with an increased percentage of blasts and/or 
cytogenetic abnormalities, in addition to del(5q). Thus, this treatment may have a particular benefit on the disease course and survival of this subset of patients who, otherwise, would have a poor prognosis. Larger studies will have to be conducted to confirm this hypothesis, however. The effect of lenalidomide has also been tested in low-risk MDS patients, without del(5q) [10]. Studies combining lenalidomide and other drugs, most notably erythropoietin, in low-risk MDS patients with anaemia [with or without del(5q)] are also in progress.

\section{Conclusion}

This case gives an important alert for clinicians to monitor MDS patients very closely when lenalidomide treatment is commenced. Lenalidomide is an effective therapeutic agent for patients with del(5q) MDS, showing an excellent effect and long-term remission in the present patient. Prior to therapy the patient was transfusion dependent, but she demonstrated severe pancytopaenia and severe chest infection when her therapy was given for only seven days. Both of these resultant conditions may have been due to the effect of lenalidomide on a large MDS clone burden. In naïve cases, MDS treatment, beginning at a low dose as $5 \mathrm{mg}$ daily instead of $10 \mathrm{mg}$ daily, may be more suitable with weekly CBC counts in order to avoid severe life threatening pancytopaenia and the need for hospitalisation in a drug meant to be used in outpatient department.

\section{Acknowledgement}

The authors acknowledge the expert technical assistance of Adriana Zameenikova from the cytogenetic laboratory, Valiyaveettil Ramani from the general haematology laboratory, Lilly Jacob, and Khoold al-Harbi from the blood bank.

\section{Disclosure Statement}

There is no conflict of interest. 


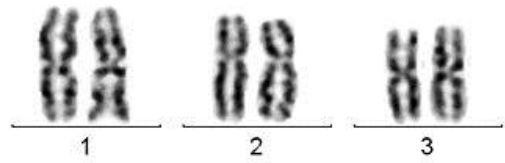

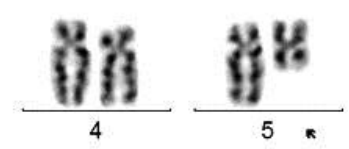

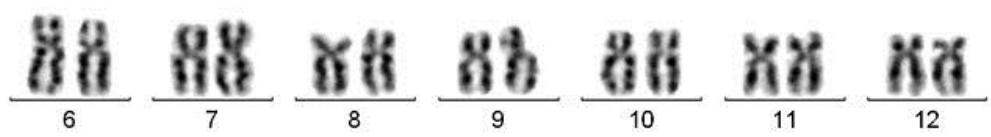

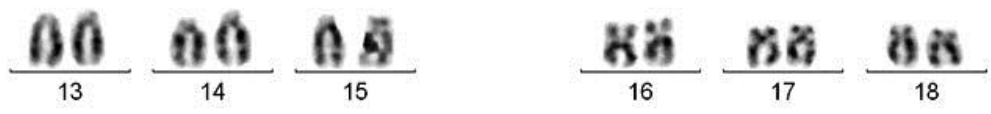

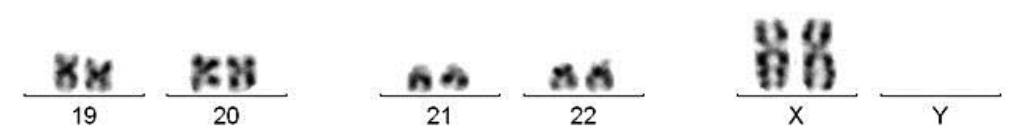

Fig. 1. Karyotype before lenalidomide treatment.
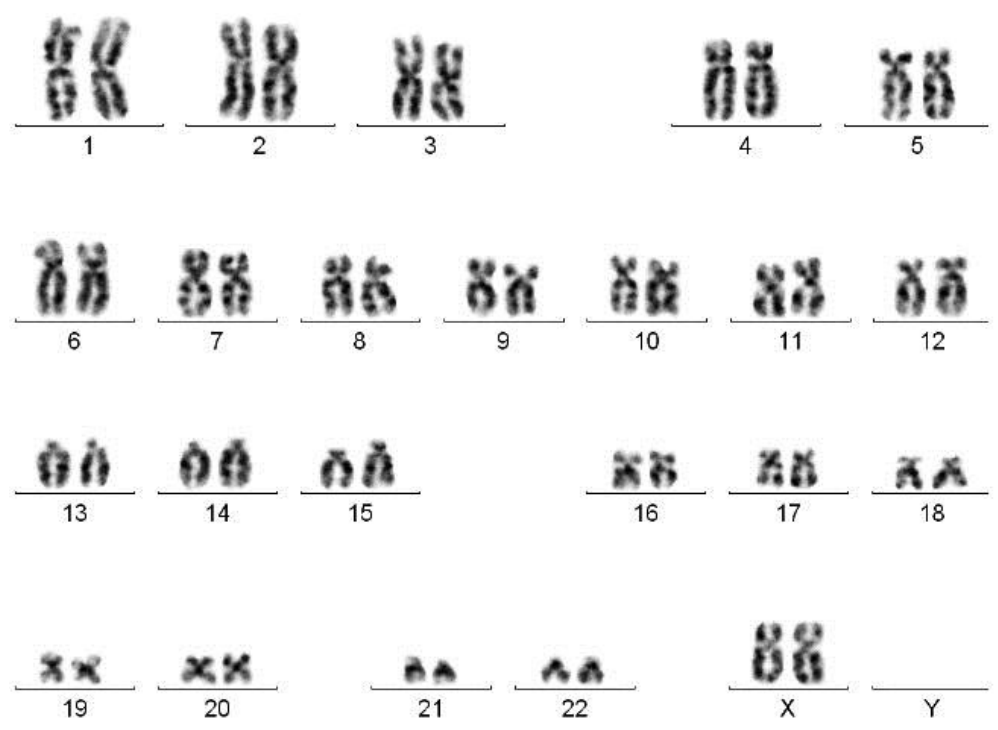

Fig. 2. Karyotype after lenalidomide treatment. 


\section{References}

1 Cheson BD: The Myelodysplastic Syndromes. The Oncologist 1997;2:28-39.

2 Warlick ED, Smith BD: Myelodysplastic syndromes: review of pathophysiology and current novel treatment approaches. Curr Cancer Drug Targets 2007;7:541-558.

3 Steensma DP, Tefferi A: The myelodysplastic syndrome(s): a perspective and review, highlighting current controversies Leuk Res 2003;27:95-120.

4 Koreth J, Antin JH: Iron overload in hematologic malignancies and outcome of allogeneic hematopoietic stem cell transplantation. Hematologica 2010;95:364-366.

-5 Fehniger TA, Byrd JC: Single-agent lenalidomide induces complete remission of acute myeloid leukemia in patients with isolated trisomy 13. Blood 2009;113:1002-1005.

6 Mufti GJ, Bennett JM, Goasguen J, et al: Diagnosis and classification of myelodysplastic syndrome: International Working Group on Morphology of myelodysplastic syndrome (IWGM-MDS) consensus proposals for the definition and enumeration of myeloblasts and ring sideroblasts. Haematologica 2008;93:1712-1717.

7 List AF, Baker AF, Green S, Bellamy W: Lenalidomide: targeted anemia therapy for myelodysplastic syndromes. Malignant Hematology Program, H Lee Moffitt Cancer Center \& Research Institute, Tampa, FL 33612, USA.

8 List AF, Baker AF, Green S, et al: Lenalidomide: targeted anemia therapy for myelodysplastic syndromes. Cancer Control 2006;13(suppl):4-11.

-9 Kiaei M, Petri S, Kipiani K, et al: Thalidomide and lenalidomide extend survival in a transgenic mouse model of amyotrophic lateral sclerosis. J Neurosci 2006;26:2467-2473.

10 Komrokji RS, List AF: Role of lenalidomide in the treatment of myelodysplastic syndromes. Semin Oncol 2011;38:648-657. 